Supporting Information

\title{
Bioinspired Engineering of Sacrificial Metal-Ligand Bonds into Elastomers with Supra Mechanical Performance and Adaptive Recovery
}

Zhenghai Tang, ${ }^{\dagger}$ Jing Huang, ${ }^{\dagger}$ Baochun Guo, ${ }^{* \dagger}$ Liqun Zhang, ${ }^{*}+\dot{*}$ and Fang Liu ${ }^{\dagger}$

${ }^{\dagger}$ Department of Polymer Materials and Engineering, South China University of Technology, Guangzhou, 510640, P. R. China.

$\$$ State Key Laboratory of Organic/Inorganic Composites, Beijing University of Chemical Technology, Beijing, 100029, P. R. China.

Table S1. Composition of the samples ${ }^{\mathrm{a}}$

\begin{tabular}{ccccc}
\hline Codes & VPR (g) & sulfur $(\mathrm{g})$ & $\begin{array}{c}\text { zinc } \\
\text { chloride/pyridine } \\
\text { mole ratio }\end{array}$ & $\begin{array}{c}\text { metal } \\
\text { ions/pyridine } \\
\text { mole ratio }\end{array}$ \\
\hline VPR- $x$ & 100 & 1.5 & $\begin{array}{c}\text { variable }(0,0.25, \\
0.33,0.5,0.67)\end{array}$ & \\
VPR-0.5-y & 100 & $\begin{array}{c}\text { variable }(0.2,0.5, \\
1.0,1.5,2.5,3.5)\end{array}$ & 0.5 & 0.5 \\
Ni(Co)-VPR & 100 & 1.5 & 0.33 \\
Fe(La)-VPR & 100 & 1.5 & & \\
\hline
\end{tabular}

${ }^{\mathrm{a}}$ rubber ingredients: zinc oxide $5 \mathrm{~g}$; stearic acid $2 \mathrm{~g}$; dibenzothiazole disulfide $0.5 \mathrm{~g}$; diphenyl guanidine $0.5 \mathrm{~g}$; tetramethyl thiuram disulfide $0.2 \mathrm{~g}$ 


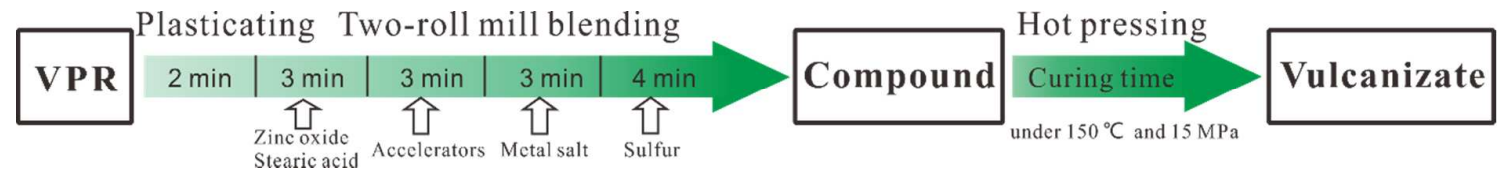

Figure S1. Flow chart for the preparation of VPR vulcanizates.

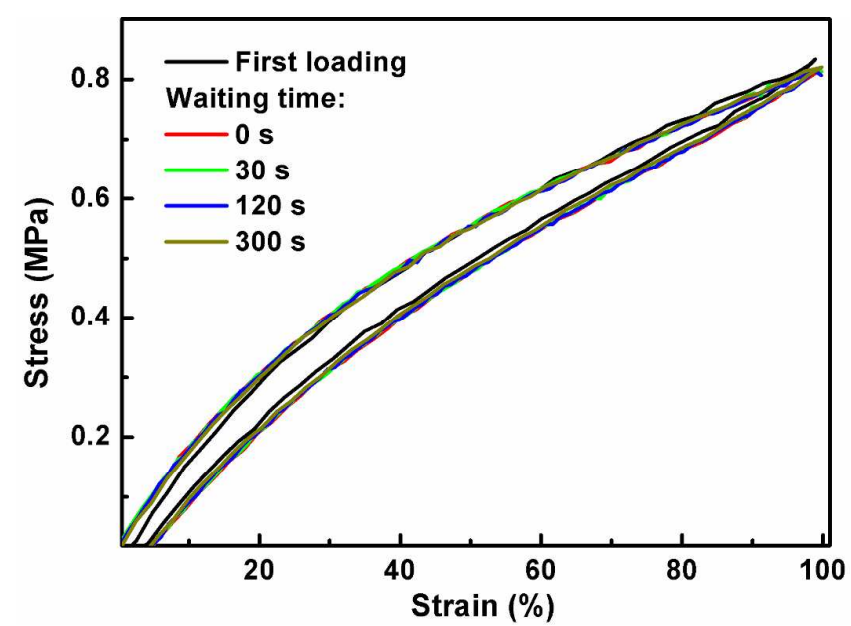

Figure S2. After the first cycle of loading and unloading, the recovery of VPR-0 after different waiting time at room temperature performed by cyclic tensile tests.
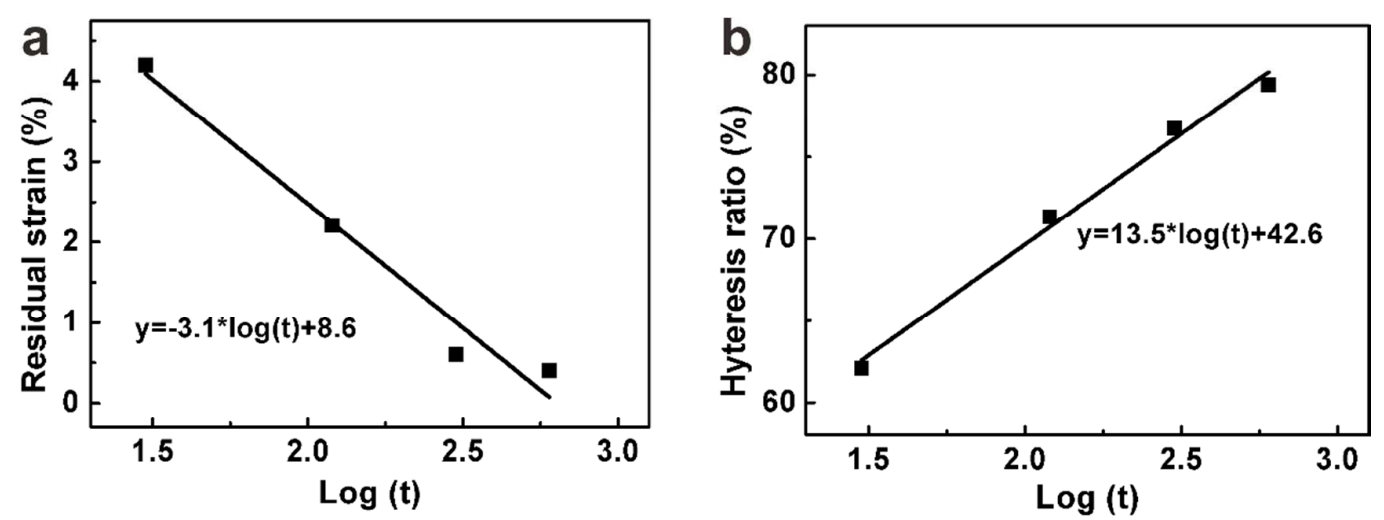

Figure S3. Plot for (a) residual strain and (b) hyteresis ratio versus the logarithm of $t$.

The straight line is a linearly fit to the data with the equation in the inset. 


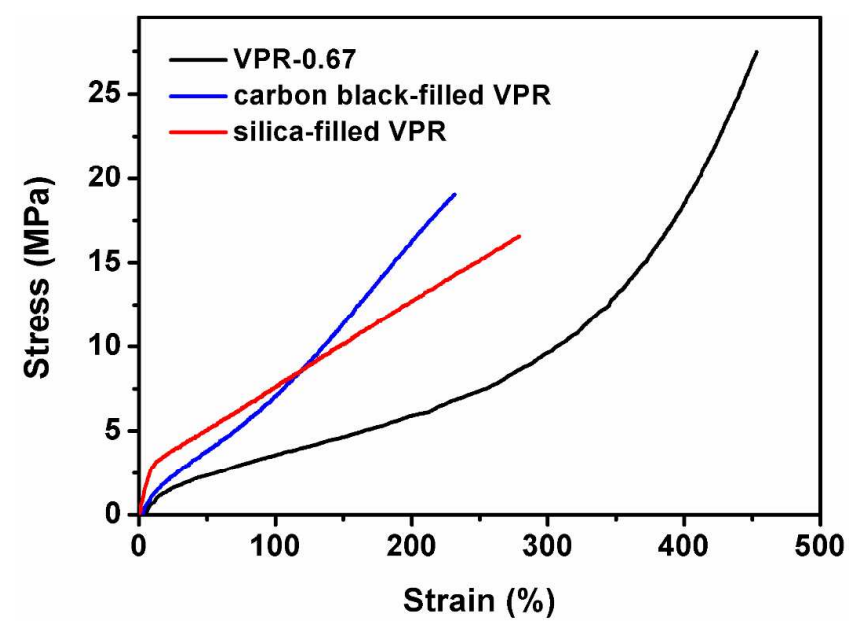

Figure S4. Comparison of the stress-strain curves for VPR-0.67, carbon black- and silica-filled VPR.

Table S2. Mechanical properties of VPR- $x$, CB- and silica-filled VPR

\begin{tabular}{|c|c|c|c|c|c|}
\hline Samples & $\begin{array}{c}\text { Young's } \\
\text { modulus } \\
(\mathrm{MPa})\end{array}$ & $\begin{array}{c}\text { Stress at } \\
100 \% \text { strain } \\
(\mathrm{MPa})\end{array}$ & $\begin{array}{c}\text { Tensile } \\
\text { strength } \\
(\mathrm{MPa})\end{array}$ & $\begin{array}{c}\text { Elongation } \\
\text { at break } \\
(\%)\end{array}$ & $\begin{array}{c}\text { Fracture } \\
\text { energy } \\
\left(\mathrm{MJ} / \mathrm{m}^{3}\right)\end{array}$ \\
\hline VPR-0 & 4.9 & 0.8 & 3.5 & 428 & 6.3 \\
\hline VPR-0.25 & 6.2 & 1.3 & 8.9 & 526 & 17.4 \\
\hline VPR-0.33 & 12.7 & 2.0 & 13.3 & 495 & 23.4 \\
\hline VPR-0.5 & 15.4 & 2.9 & 20.4 & 453 & 30.6 \\
\hline VPR-0.67 & 19.8 & 3.7 & 27.8 & 454 & 38.7 \\
\hline $\begin{array}{c}\text { carbon } \\
\text { black-filled } \\
\text { VPR }\end{array}$ & 35.1 & 6.9 & 17.7 & 216 & 20.1 \\
\hline $\begin{array}{c}\text { silica-filled } \\
\text { VPR }\end{array}$ & 38.4 & 7.2 & 17.5 & 304 & 21.4 \\
\hline
\end{tabular}


Table S3. Mechanical properties of VPR-0.5-y with different sulfur content. The mole ratio of $\mathrm{ZnCl}_{2} / \mathrm{VP}$ is 0.5 .

\begin{tabular}{|c|c|c|c|c|c|}
\hline Samples & $\begin{array}{c}\text { Young's } \\
\text { modulus } \\
(\mathrm{MPa})\end{array}$ & $\begin{array}{c}\text { Stress at } \\
100 \% \text { strain } \\
(\mathrm{MPa})\end{array}$ & $\begin{array}{c}\text { Tensile } \\
\text { strength } \\
(\mathrm{MPa})\end{array}$ & $\begin{array}{c}\text { Elongation } \\
\text { at break } \\
(\%)\end{array}$ & $\begin{array}{c}\text { Fracture } \\
\text { energy } \\
\left(\mathrm{MJ} / \mathrm{m}^{3}\right)\end{array}$ \\
\hline VPR-0.5-0.2 & 7.6 & 1.9 & 24.2 & 615 & 44.3 \\
\hline VPR-0.5-0.5 & 9.3 & 2.1 & 25.6 & 579 & 37.3 \\
\hline VPR-0.5-1.0 & 13.1 & 2.9 & 22.6 & 474 & 35.5 \\
\hline VPR-0.5-1.5 & 15.4 & 2.9 & 20.4 & 453 & 31.5 \\
\hline VPR-0.5-2.5 & 23.9 & 5.1 & 18.8 & 323 & 28.8 \\
\hline VPR-0.5-3.5 & 27.8 & 6.2 & 18.7 & 273 & 23.6 \\
\hline
\end{tabular}

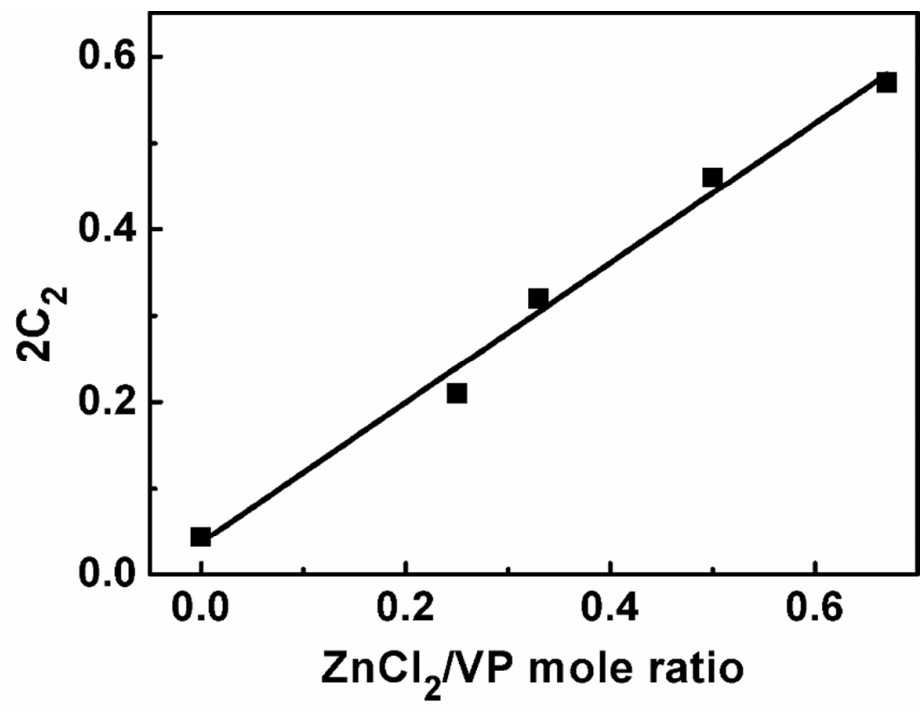

Figure S5. Plot for $2 \mathrm{C}_{2}$ versus $\mathrm{ZnCl}_{2} / \mathrm{VP}$ mole ratio. 


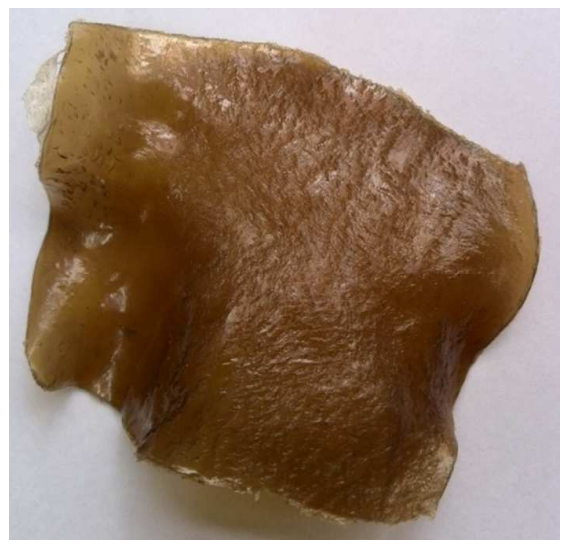

Figure S6. Appearance of the VPR-0.5-0 without sulfur.

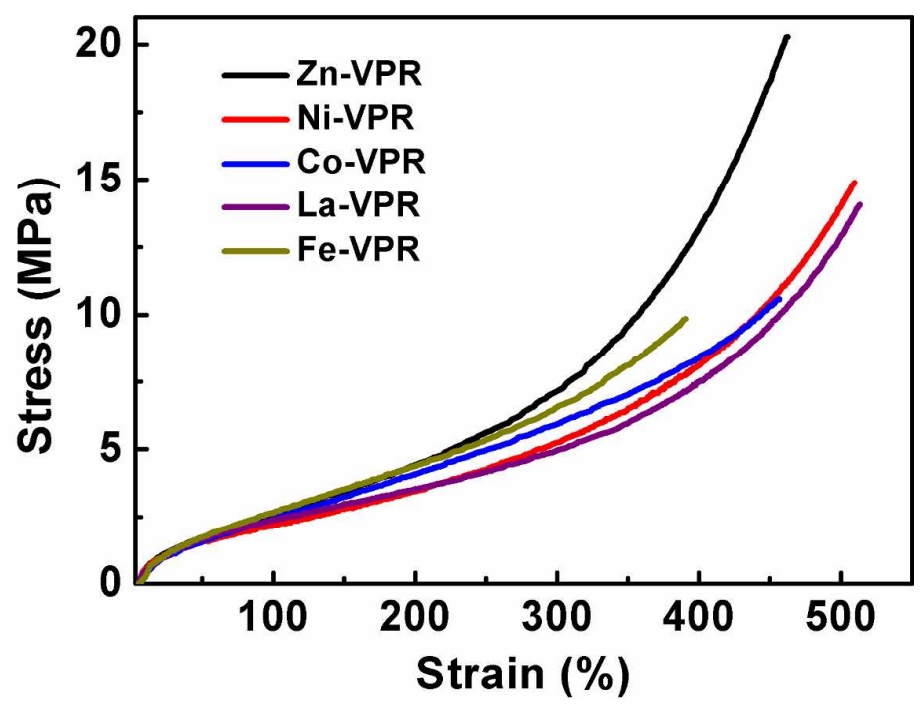

Figure S7. Typical stress-strain curves of VPR by incorporating different metal ions. 


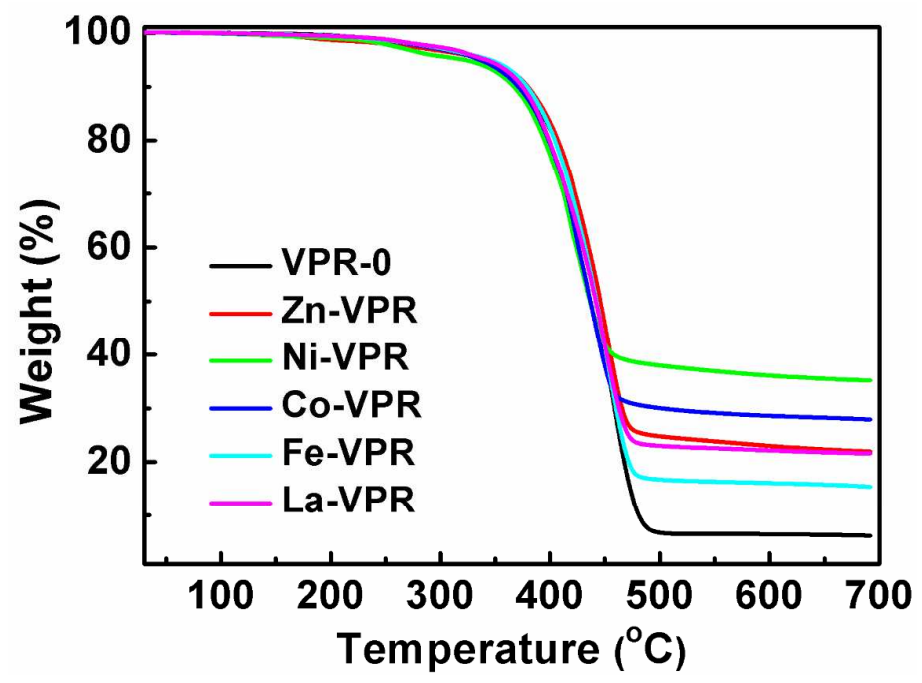

Figure S8. TGA curves of neat VPR and VPR samples with various metal ions.

Table S4. Mechanical properties of VPR using different metal ions.

\begin{tabular}{|c|c|c|c|c|c|}
\hline Samples & $\begin{array}{c}\text { Young's } \\
\text { modulus } \\
(\mathrm{MPa})\end{array}$ & $\begin{array}{c}\text { Stress at } \\
300 \% \text { strain } \\
(\mathrm{MPa})\end{array}$ & $\begin{array}{c}\text { Tensile } \\
\text { strength } \\
(\mathrm{MPa})\end{array}$ & $\begin{array}{c}\text { Elongation } \\
\text { at break } \\
(\%)\end{array}$ & $\begin{array}{c}\text { Fracture } \\
\text { energy } \\
\left(\mathrm{MJ} / \mathrm{m}^{3}\right)\end{array}$ \\
\hline Zn-VPR & 15.4 & 7.6 & 20.4 & 453 & 31.5 \\
\hline Ni-VPR & 9.0 & 5.2 & 13.9 & 514 & 27.1 \\
\hline Co-VPR & 11.4 & 5.6 & 11.6 & 486 & 22.1 \\
\hline La-VPR & 6.7 & 4.9 & 14.1 & 513 & 26.3 \\
\hline Fe-VPR & 10.5 & 6.6 & 9.9 & 391 & 17.8 \\
\hline
\end{tabular}




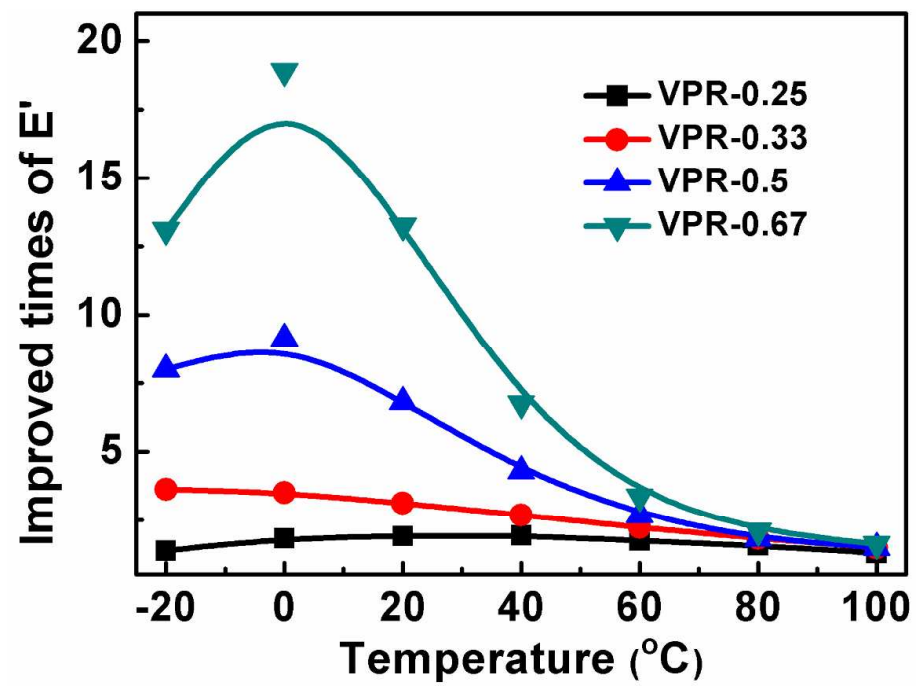

Figure S9. The E' of VPR- $x$ is normalized to that of VPR-0 at different temperature. 